\title{
Voxel-wise meta-analysis of structural changes in gray matter of Parkinson's disease patients with mild cognitive impairment
}

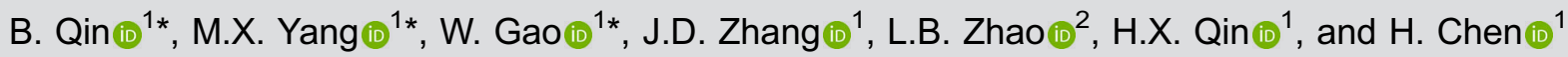 \\ ${ }^{1}$ Department of Neurology, Affiliated Liuzhou People's Hospital of Guangxi University of Science and Technology/Liuzhou People's \\ Hospital, Liuzhou, Guangxi, China \\ ${ }^{2}$ Department of Neurology, Yongchuan Hospital of Chongqing Medical University, Chongqing, China
}

\begin{abstract}
Evidence from previous voxel-based morphometry (VBM) studies indicates that widespread brain regions are involved in Parkinson's disease with mild cognitive impairment (PD-MCI). However, the spatial localization reported for gray matter (GM) abnormalities is heterogeneous. The aim of the present study was to quantitatively integrate studies on GM abnormalities observed in PD-MCI in order to determine whether a pattern exists. Eligible whole-brain VBM studies were identified by a systematic search of articles in PubMed and EMBASE databases spanning from 1995 to January 1, 2019. A meta-analysis was performed to investigate regional GM abnormalities in PD-MCl. The anisotropic effect size version of seed-based d mapping (AES-SDM) meta-analysis was conducted to explore the GMV differences of PD-MCl compared with PD patients with normal cognitive function (PD-NC). A total of 12 studies comprising 243 PD-MCl patients and 326 PD-NC were included in the metaanalysis. PD-MCl patients showed a robust GM decrease in the left insula and left superior temporal gyrus. Moreover, meta-regression analysis demonstrated that age, PD duration and stage, and Unified Parkinson's Disease Rating Scale III and Mini-Mental State Examination scores might be partly correlated with the GM abnormalities observed in PD-MCI patients. The convergent findings of this quantitative meta-analysis revealed a characteristic neuroanatomical pattern in PD-MCl. The findings provide some evidence that $\mathrm{MCl}$ in PD may result in the breakdown of the insula and temporal gyrus, which may serve as specific regions of interest for further investigations.
\end{abstract}

Key words: Voxel-based morphometry; Gray matter; Meta-analysis; Parkinson's disease; Mild cognitive impairment

\section{Introduction}

Parkinson's disease (PD) is the most common chronic neurodegenerative disease and may lead to mild cognitive impairment $(\mathrm{MCl})$ and dementia (1). The estimated prevalence of PD with dementia (PDD) ranges from 10 to $30 \%$, and the risk of dementia is almost six times higher in PD patients than in the general population $(2,3)$. PD patients without dementia but with mild cognitive impairment (i.e., PD-MCl) are at increased risk of developing dementia (4-6). As in Alzheimer's disease, patients with $\mathrm{PD}-\mathrm{MCl}$ have an increased risk of developing dementia compared with those without the condition $(5,6)$. Thus, cognitive dysfunction is a common and debilitating feature of $\mathrm{PD}$; moreover, $\mathrm{MCl}$ is an early manifestation of dementia in PD patients. However, the factors contributing to cognitive dysfunction in PD are not completely understood.
Neuroimaging is useful for investigating brain structural features of PD-MCl patients. Voxel-based morphometry (VBM) is a fully automated, quantitative magnetic resonance imaging (MRI)-associated processing method for detecting subtle morphological and neuropathological changes in the whole brain and is used to quantify changes in gray matter volume (GMV) in neuropsychiatric and neurological disorders such as PD and PDD (7). VBM has also been applied to the investigation of GM atrophy in PD-MCl; however, the findings have been contradictory. For instance, GM loss has been reported in cortical and subcortical regions mainly in the left middle frontal gyrus, precentral gyrus, left superior temporal lobe, and right inferior temporal lobe of PD-MCI patients (8), whereas another study found no differences in GM volume between PD patients with normal cognitive function (PD-NC) and

Correspondence: H. Chen: <Izrmyych@126.com>

*These authors contributed equally to this study. 
those with PD-MCl (9). Furthermore, meta-analyses comparing $\mathrm{PD}-\mathrm{MCl}$ and $\mathrm{PD}-\mathrm{NC}$ patients found $\mathrm{GM}$ atrophy in the left superior temporal lobe, left insula, and left superior frontal lobe, which was linked to cognitive impairment (10), as well as reduced GMV in the left insula and left superior frontal lobe in PD-MCl patients (11). However, other investigations failed to detect any GM atrophy related to cognitive impairment $(12,13)$. These reported discrepancies could be due to the limited number of studies included in previous meta-analyses; recently, there have been more studies using VBM to investigate changes in brain structure in PD-MCl patients.

Anisotropic effect size-based signed differential mapping (AES-SDM) is a newly developed meta-analysis tool for neuroimaging data that has been applied to examinations of multiple disorders, including obsessive-compulsive disorder and bipolar disorder, and others (14-16), as well as to VBM studies on GMV in PD and other neurological diseases $(17,18)$. AES-SDM allows exhaustive and accurate meta-analysis by employing standard effect size and variance-based calculations in addition to combining both peak coordinates and statistical parametric maps. It also shows good overlap with pool analysis and has adequate sensitivity and excellent control of false positives (19). In order to establish a consistent and reliable GM map in $\mathrm{PD}-\mathrm{MCl}$ patients, here we carried out a comprehensive meta-analysis of recent VBM studies using AES-SDM. Our findings provided insights into the neuroanatomical substrates and pathophysiological mechanisms of PD-MCI that could improve diagnosis and treatment.

\section{Material and Methods}

\section{Literature search}

Systematic and comprehensive searches of articles in PubMed and EMBASE databases published between 1995 and January 1, 2019 were performed using the following keywords: "voxel" or "VBM or morphometry or gray matter or grey matter" AND "cognitive impairment or mci or cognitive decline or cognition" AND "Parkinson's disease or Parkinson OR PD". A manual search of the reference lists of relevant articles was performed to identify additional potential studies.

\section{Study selection and data extraction}

Studies were included in the meta-analysis if they met the following criteria: 1) the patient group included PD-MCI or PD-NC patients; 2) VBM studies reported a voxel-wise comparison of GM density or volume between PD-MCI and PD-NC patients; 3) whole-brain results in threedimensional coordinates $(x, y, z)$ of changes in standard stereotactic space (Talairach or Montreal Neurological Institute) were reported; 4) significance thresholds corrected for multiple comparisons or uncorrected with spatial extent thresholds were used; 5) the sample size in each group was more than 5; and 6) studies were published in
English as a peer-reviewed article (not as a letter or abstract). The exclusion criteria were as follows: 1) sufficient data for the meta-analysis could not be obtained even after corresponding with the authors by email; 2) findings were based solely on small volume correction; and 3) region of interest methods were used. Three researchers independently performed the study selection and data extraction and any discrepancies were resolved by consensus through discussion. The study followed the guidelines of preferred reporting items for systematic reviews and meta-analyses (Figure 1).

\section{Statistical analyses and data preparation}

Regional differences in GM changes between PD-MCI and PD-NC patients were analyzed using AES-SDM software (version 4.31, http://www.sdmproject.com), a new version of SDM that has been described in detail elsewhere $(19,20)$. The analytical processes were referred to the AES-SDM tutorial and guidelines. First, coordinates of cluster peaks and effect-sizes (derived from e.g., $\mathrm{t}$-values, or equivalently from $\mathrm{P}$-values or z-scores) were extracted from each data set according to AES-SDM inclusion criteria. An effect size signed map of the differences in GM was separately recreated for each study. Full effect sizes were assigned to peaks (positive for GM increases and negative for decreases), and decreasing effect sizes were assigned to voxels that showed a lower correlation with the peak (20). Second, a mean map was obtained by voxel-wise calculation of the random-effects mean of study maps that were weighted by the sample size and variance of each study. Any potential heterogeneity associated with the main coordinates were also assessed by AES-SDM. Finally, a more stringent significance threshold was set to uncorrected $\mathrm{P}<0.001$ (empirically equivalent to $\mathrm{P}<0.05$, corrected) and a cluster-level threshold of 10 voxels (21). Additional jackknife sensitivity analyses were also carried out by excluding one sample at a time in order to evaluate the robustness of the results across different studies.

Meta-regression analysis was performed to determine the correlation between clinical variables (e.g., age, disease duration, education level, Mini-Mental State Examination [MMSE] score, and Unified Parkinson's Disease Rating Scale [UPDRS]-III score) and GMV changes that could contribute to the heterogeneity of the results. We used a voxel threshold of $\mathrm{P}<0.0005$ and cluster extent $=10$ voxels in the meta-regression analyses. Maps showing statistical significance were visualized using MRIcron (https://www.mccauslandcenter.sc.edu/crnl/mricro).

\section{Results}

After an initial assessment of 1069 relevant studies identified with our search strategy, 20 VBM studies of GM changes in PD-MCl patients were identified. Eight of these 


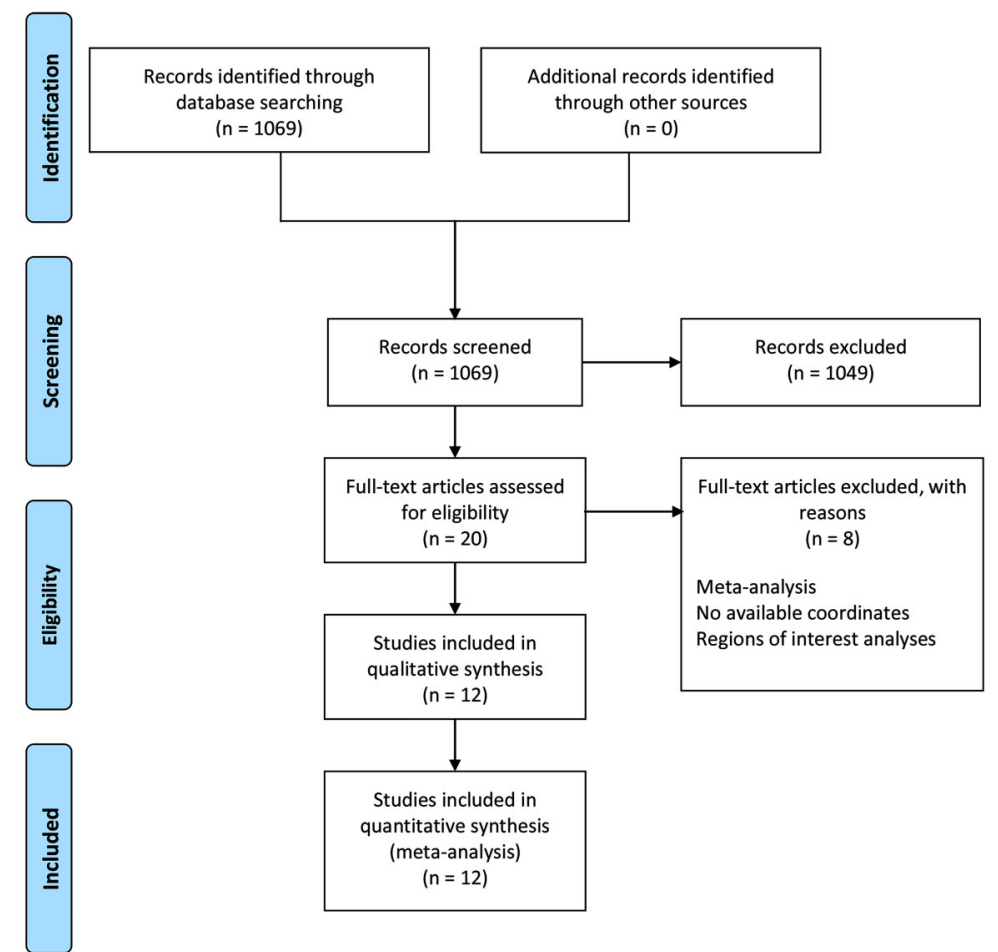

Figure 1. Search strategy used for the inclusion of studies that were considered for the meta-analysis.

were excluded for the following reasons: 1) use of a regions of interest analyses; 2) no available coordinates; and 3) published as a meta-analysis. Thus, 12 studies comprising $243 \mathrm{PD}-\mathrm{MCl}$ and $326 \mathrm{PD}-\mathrm{NC}$ patients were ultimately included in the meta-analysis $(8,9,11-13,22-$ 28). Demographic and clinical characteristics for each study that met the inclusion criteria and neuroimaging parameters are outlined in Supplementary Table S1.

\section{Pooled meta-analysis of regional GM changes}

Voxel-based AES-SDM meta-analysis identified the temporal-insular-frontal lobe as the primary site of GM abnormalities associated with PD-MCl (Figure 2). Regions with significantly less GM were the left insula, left superior temporal gyrus, and left inferior frontal gyrus. Peak coordinates and cluster breakdowns are shown in Table 1. There were no regions showing increased GMV in PD$\mathrm{MCl}$ compared to PD-NC patients.

\section{Jackknife sensitivity and heterogeneity analyses}

Systematic whole-brain jackknife sensitivity analysis of the $\mathrm{PD}-\mathrm{MCl}$ findings revealed a significant reduction in GM in the left insula in 11 of 12 datasets. GM abnormalities in the left superior temporal gyrus were highly replicable and remained significant in 10 of 12 datasets (Table 1). None of the effect size differences in peak coordinates showed significant heterogeneity.

\section{Meta-regression}

Meta-regression analyses revealed that age was negatively associated with GMV in the left rolandic operculum and that longer disease duration was correlated with greater $\mathrm{GM}$ atrophy in the right inferior temporal gyrus, left temporal pole, superior temporal gyrus, and left middle temporal gyrus. Additionally, higher UPDRS-III score and Hoehn and Yahr stage were correlated with greater GM atrophy in the left inferior temporal gyrus, right inferior temporal gyrus, left inferior frontal gyrus, and right middle frontal gyrus. There was no significant association between GMV reduction and education level or MMSE scores in PD-MCl patients. Detailed information is summarized in Table 2.

\section{Discussion}

Our modified and comprehensive meta-analysis of 12 case-control VBM studies by AES-SDM showed that $\mathrm{PD}-\mathrm{MCl}$ patients had less $\mathrm{GM}$ in the left superior temporal gyrus, left inferior frontal gyrus, and left insula. Moreover, the meta-regression analysis revealed that age, disease duration, UPDRS-III score, and Hoehn and Yahr stage were partly correlated with GM abnormalities in this group.

One of the most important findings of this study was that PD-MCl patients consistently showed significant 


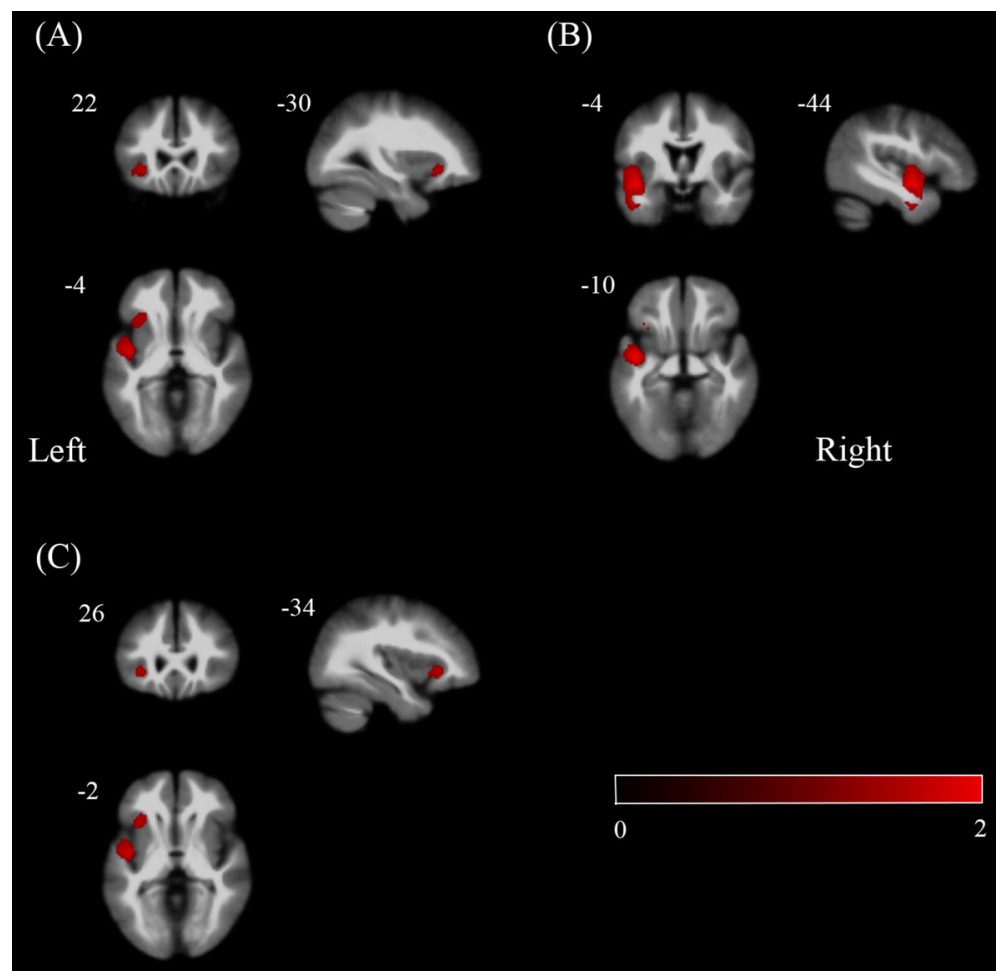

Figure 2. Areas with lower gray matter in Parkinson's disease with mild cognitive impairment relative to Parkinson's disease with normal cognitive function are displayed in red in (A) left insula, (B) left superior temporal gyrus, and (C) left inferior frontal gyrus. The color bar indicates the maximum and the minimum seed-based $d$ mapping- $Z$ values.

Table 1. Clusters of gray matter volume reductions in patients with Parkinson's disease with mild cognitive impairment compared with Parkinson's disease with normal cognitive function.

\begin{tabular}{lcccccc}
\hline Regions & $\begin{array}{c}\text { No. of } \\
\text { voxels }\end{array}$ & $\begin{array}{c}\text { Maximum MNI } \\
\text { coordinates }\end{array}$ & SDM-Z value & P value & $\begin{array}{c}\text { Jackknife sensitivity } \\
\text { analysis }\end{array}$ & $P$ (Egger's test) \\
\hline Left insula & 100 & $-30,22,-4$ & -1.723 & 0.000099063 & 11 out of 12 & 0.250 \\
Left superior temporal gyrus & 914 & $-44,-4,-10$ & -2.176 & 0.000000536 & 10 out of 12 & 0.325 \\
Left inferior frontal gyrus & 147 & $-34,26,-2$ & -1.726 & 0.000096500 & 6 out of 12 & 0.250 \\
\hline
\end{tabular}

MNI: Montreal Neurological Institute; SDM: seed-based d mapping.

reductions in GM in the left superior temporal gyrus, left inferior frontal gyrus, and left insula compared to PD-NC patients. The results of the meta-regression analysis indicated that decreased $\mathrm{GM}$ in these regions in PD-MCI patients was associated with older age, longer disease duration, and higher UPDRS-III score and Hoehn and Yahr stage. Imaging studies of cognitive impairment have shown GM reduction in fronto-temporal and posterior cortical areas $(8,13,28,29-31)$. Importantly, GM abnormalities in the left temporal area were associated with impaired executive function, attention, and memory; those in the left frontal area were associated with impaired attention; and those in the left insular area were associated with impaired executive function, attention, and language abilities. This seems to suggest a possible relationship between cognitive decline in PD and structural decline in these brain regions, which is supported by the neuroanatomical abnormalities in the left superior temporal gyrus, left inferior frontal gyrus, and left insula identified in our meta-analysis. There is also some evidence that the frontal-limbic-temporal region (mainly the left temporal gyrus, left frontal gyrus, and left insula) is a potential therapeutic target in PD-MCl, although additional studies are needed to determine whether treatment reverses these structural changes and whether this morphometric pattern can serve as a prognostic biomarker. 
Table 2. Meta-regression analyses of demographic and clinical variables in Parkinson's disease with mild cognitive impairment patients.

\begin{tabular}{|c|c|c|c|c|}
\hline Variables/Anatomical regions & Peak MNI coordinates $(x, y, z)$ & Number of voxels & SDM-Z value & $P$ value \\
\hline \multicolumn{5}{|l|}{ Age } \\
\hline Left rolandic operculum & $-50,0,4$ & 1053 & -2.401 & 0.000030994 \\
\hline \multicolumn{5}{|l|}{ PD duration } \\
\hline Right inferior temporal gyrus & $56,-20,-26$ & 80 & -1.415 & 0.000141382 \\
\hline Left temporal pole, superior temporal gyrus & $-30,20,-30$ & 45 & -1.518 & 0.000053644 \\
\hline Left middle temporal gyrus & $-62,-24,2$ & 15 & -1.361 & 0.000374138 \\
\hline \multicolumn{5}{|l|}{ UPDRS-III score } \\
\hline Left inferior temporal gyrus & $-46,-8,-40$ & 106 & -1.534 & 0.000009835 \\
\hline Right inferior temporal gyrus & $46,-6,-40$ & 39 & -1.507 & 0.000207961 \\
\hline Left inferior frontal gyrus & $-40,34,16$ & 30 & -1.513 & 0.000163078 \\
\hline \multicolumn{5}{|l|}{ Hoehn and Yahr stage } \\
\hline Left inferior temporal gyrus & $-46,-10,-36$ & 88 & -1.386 & 0.000248730 \\
\hline Right inferior temporal gyrus & $48,-10,-38$ & 85 & -1.507 & 0.000128508 \\
\hline Left inferior frontal gyrus & $-40,36,14$ & 22 & -1.318 & 0.000505269 \\
\hline Right middle frontal gyrus & $38,44,12$ & 17 & -1.317 & 0.000588357 \\
\hline Education level & - & - & - & - \\
\hline MMSE scores & - & - & - & - \\
\hline
\end{tabular}

MNI: Montreal Neurological Institute; SDM: seed-based d mapping; PD: Parkinson's disease; UPDRS-III: Unified Parkinson's Disease Rating Scale: Part III; MMSE: mini-mental state examination.

The insula and temporal gyrus constitute the ventral stream of fronto-parietal pathways that play an important role in working memory and executive function (32). This stream is activated during attention shifting, interference resolution, and strategic organization (33); thus, atrophy of the insula and temporal gyrus can negatively impact frontal lobe-based cognitive performance. Our finding of a significant association between insula-temporal gyrus atrophy and deficits in executive function and attention in PD-MCl patients suggests early disruption of the ventral fronto-parietal pathway as a likely mechanism for the cognitive impairment observed in PD. Our results were consistent with those reported in a previous meta-analysis showing a decrease in signal intensity in the left temporal lobe extending to the left insula and left frontal lobe in patients with $\mathrm{MCl}$ compared with those without cognitive deficits (10). However, our meta-analysis included more studies than previous ones, which increased the statistical power. In addition, resting-state functional MRI revealed a reduced connectivity specifically in the default mode network (left precuneus, right median cingulate gyrus, left superior frontal gyrus, and right precentral gyrus) in PD-MCl patients (34). Interestingly, another recent metaanalysis found differences in several brain regions (left insula, bilateral dorsolateral prefrontal cortex, left angular gyrus, midcingulate cortex, and right supramarginal gyrus) between PD-MCI and PD-NC patients (35), which was not entirely consistent with our findings. This could be due to the fact that the earlier meta-analysis was biased as it pooled multiple studies that employed both VBM and cortical thickness measures, which may have reduced the sensitivity of detecting structural changes in subcortical regions such as the basal ganglia (35).

Interestingly, we did not consistently observe GM abnormalities in the hippocampus, which is a hallmark of Alzheimer's disease (36) even though over half of PD patients exhibit Alzheimer's disease-related neuropathologic changes in the hippocampus that are detectable by autopsy (37). Hippocampal GMV reduction has been linked to hippocampal neuron atrophy in the $\mathrm{MCl}$ stage of PD (38). This discrepancy may be attributed to the limited number of studies that were included in our meta-analysis, sample features, or methodological factors. It has been reported that neurodegeneration in PD patients begins in the head and spreads to the tail of the hippocampus (39). However, the results of our study indicated that hippocampal atrophy was not closely associated with the initial stages of cognitive dysfunction in PD.

Our study had several limitations. First, the sample sizes of included studies were relatively small, and therefore the results must be interpreted with caution. On the other hand, a large number of studies were included and our findings can be considered robust because nearly all of the studies contributed to the results. Second, the heterogeneity of VBM study methodology including different preprocessing protocols and statistical thresholding methods may have influenced the results. Third, the results of the meta-regression analysis also found longer disease duration was correlated with greater GM atrophy in the left superior temporal gyrus. This seems to suggest the degenerative course of the disease in PD also may have occurred along grey matter loss in 
the left superior temporal gyrus, which may have led to less accurate results. However, a recent meta-analysis found that GM reductions were detected in the right temporal lobe, right rolandic operculum, right amygdala, right angular gyrus, right middle occipital gyrus, right fusiform gyrus, left superior frontal gyrus, bilateral anterior cingulate/paracingulate gyri, bilateral insula, and bilateral striatum in the PD patients compared with the healthy controls. Regions with significantly less GM were the left insula, left superior temporal gyrus, and left inferior frontal gyrus. No significant regional GM atrophy in left superior temporal gyrus was detected in the PD patients (40).

In conclusion, the present voxel-wise meta-analysis revealed that GM was reduced in the left insula and left superior temporal gyrus of $\mathrm{PD}-\mathrm{MCl}$ patients compared with PD-NC patients. Furthermore, we found that age, disease duration, UPDRS-III scores, and Hoehn and Yahr

\section{References}

1. Schapira AH. The measurement and importance of nonmotor symptoms in Parkinson disease. Eur J Neurol 2015; 22: 2-3, doi: 10.1111/ene.12523.

2. Aarsland D, Tandberg E, Larsen JP, Cummings JL. Frequency of dementia in Parkinson disease. Arch Neurol 1996; 53: 538-542, doi: 10.1001/archneur.1996.00550060 082020.

3. Aarsland D, Andersen K, Larsen JP, Lolk A, Nielsen H, KraghSorensen P. Risk of dementia in Parkinson's disease: a community based, prospective study. Neurology 2001; 56 : 730-736, doi: 10.1212/WNL.56.6.730.

4. Caviness JN, Driver-Dunckley E, Connor DJ, Sabbagh MN, Hentz JG, Noble B, et al. Defining mild cognitive impairment in Parkinson's disease. Mov Disord 2007; 22: 1272-1277, doi: $10.1002 / \mathrm{mds} .21453$.

5. Janvin CC, Larsen JP, Aarsland D, Hugdahl K. Subtypes of mild cognitive impairment in Parkinson's disease: progression to dementia. Mov Disord 2006; 21: 1343-1349, doi: $10.1002 /$ mds.20974.

6. Williams-Gray $\mathrm{CH}$, Evans JR, Goris A, Foltynie T, Ban M, Robbins TW, et al. The distinct cognitive syndromes of Parkinson's disease: 5 year follow-up of the CamPalGN cohort. Brain 2009; 132: 2958-2969, doi: 10.1093/brain/ awp245.

7. Pan PL, Shi HC, Zhong JG, Xiao PR, Shen Y, Wu LJ, et al. Gray matter atrophy in Parkinson's disease with dementia: evidence from meta-analysis of voxel-based morphometry studies. Neurol Sci 2013; 34: 613-619, doi: 10.1007/ s10072-012-1250-3.

8. Beyer MK, Janvin CC, Larsen JP, Aarsland D. A magnetic resonance imaging study of patients with Parkinson's disease with mild cognitive impairment and dementia using voxel-based morphometry. J Neurol Neurosurg Psychiatry 2007; 78: 254-259, doi: 10.1136/jnnp.2006.093849.

9. Dalaker TO, Zivadinov R, Larsen JP, Beyer MK, Cox JL, Alves G, et al. Gray matter correlations of cognition in incident Parkinson's disease. Mov Disord 2010; 25: 629633, doi: $10.1002 / \mathrm{mds} .22867$. stage were partly correlated with GM abnormalities in PD$\mathrm{MCl}$ patients. These findings may provide a basis for investigations on the mechanisms underlying changes in the visual cortex that are associated with cognitive decline in PD. Future longitudinal and multicenter MRI studies should investigate whether this brain morphometric pattern can serve as a useful target and a prognostic marker for $\mathrm{PD}-\mathrm{MCl}$ diagnosing and treatment.

\section{Supplementary Material}

Click here to view [pdf].

\section{Acknowledgments}

We thank Charlesworth Author Services (https://www. cwauthors.com.cn/) for editing the English text of a draft of this manuscript.

10. Xu Y, Yang J, Hu X, Shang $\mathrm{H}$. Voxel-based meta-analysis of gray matter volume reductions associated with cognitive impairment in Parkinson's disease. J Neurol 2016; 263: 1178-1187, doi: 10.1007/s00415-016-8122-3.

11. Mak E, Zhou J, Tan LC, Au WL, Sitoh YY, Kandiah N. Cognitive deficits in mild Parkinson's disease are associated with distinct areas of grey matter atrophy. J Neurol Neurosurg Psychiatry 2014; 85: 576-580, doi: 10.1136/jnnp2013-305805.

12. Chen B, Wang S, Sun W, Shang X, Liu H, Liu G, et al. Functional and structural changes in gray matter of parkinson's disease patients with mild cognitive impairment. Eur J Radiol 2017; 93: 16-23, doi: 10.1016/j.ejrad.2017. 05.018.

13. Melzer TR, Watts R, MacAskill MR, Pitcher TL, Livingston L, Keenan RJ, et al. Grey matter atrophy in cognitively impaired Parkinson's disease. J Neurol Neurosurg Psychiatry 2012; 83: 188-194, doi: 10.1136/jnnp-2011-300828.

14. Radua J, Mataix-Cols D. Voxel-wise meta-analysis of grey matter changes in obsessive-compulsive disorder. $\mathrm{Br} J$ Psychiatry 2009; 195: 393-402, doi: 10.1192/bjp.bp.108. 055046.

15. Bora E, Fornito A, Yucel M, Pantelis C. Voxelwise metaanalysis of gray matter abnormalities in bipolar disorder. Biol Psychiatry 2010; 67: 1097-1105, doi: 10.1016/j.biopsych. 2010.01.020.

16. Via E, Radua J, Cardoner N, Happe F, Mataix-Cols D. Metaanalysis of gray matter abnormalities in autism spectrum disorder: should Asperger disorder be subsumed under a broader umbrella of autistic spectrum disorder? Arch Gen Psychiatry 2011; 68: 409-418, doi: 10.1001/archgenpsy chiatry.2011.27.

17. Pan PL, Song W, Shang HF. Voxel-wise meta-analysis of gray matter abnormalities in idiopathic Parkinson's disease. Eur J Neurol 2012; 19: 199-206, doi: 10.1111/j.1468-1331. 2011.03474.x.

18. Zhong J, Pan P, Dai Z, Shi H. Voxelwise meta-analysis of gray matter abnormalities in dementia with Lewy bodies. 
Eur J Radiol 2014; 83: 1870-1874, doi: 10.1016/j.ejrad. 2014.06.014.

19. Radua J, Mataix-Cols D, Phillips ML, El-Hage W, Kronhaus $\mathrm{DM}$, Cardoner $\mathrm{N}$, et al. A new meta-analytic method for neuroimaging studies that combines reported peak coordinates and statistical parametric maps. Eur Psychiatry 2011; 27: 605-611, doi: 10.1016/j.eurpsy.2011.04.001.

20. Radua J, Rubia K, Canales EJ, Pomarol-Clotet E, FusarPoli $P$, Mataix-Cols D. Anisotropic kernels for coordinate-based meta-analyses of neuroimaging studies. Front Psychiatry 2014; 5: 13, doi: 10.3389/fpsyt.2014.00013.

21. Pan $\mathrm{P}$, Shi H, Zhong J, Xiao P, Shen $\mathrm{Y}, \mathrm{Wu} \mathrm{L}$, et al. Chronic smoking and brain gray matter changes: evidence from meta-analysis of voxel-based morphometry studies. Neurol Sci 2013; 34: 813-817, doi: 10.1007/s10072-012-1256-x.

22. Agosta F, Canu E, Stefanova E, Sarro L, Tomić A, Špica V, et al. Mild cognitive impairment in Parkinson's disease is associated with a distributed pattern of brain white matter damage. Hum Brain Mapp 2014; 35: 1921-1929, doi: 10.1002/hbm.22302.

23. Chen FX, Kang DZ, Chen FY, Liu Y, Wu G, Li X, et al. Gray matter atrophy associated with mild cognitive impairment in Parkinson's disease. Neurosci Lett 2016; 617: 160-165, doi: 10.1016/j.neulet.2015.12.055.

24. Gao Y, Nie K, Huang B, Mei M, Guo M, Xie S, et al. Changes of brain structure in Parkinson's disease patients with mild cognitive impairment analyzed via VBM technology. Neurosci Lett 2017; 658: 121-132, doi: 10.1016/j.neulet.2017. 08.028.

25. Hu XF, Zhang JQ, Zhang YL, Wu Y, Chen LH, Wang J. Mild cognitive impairment in patients with Parkinson's disease: A voxel-based morphometry analysis of gray matter [in Chinese]. Chinese Journal of Medical Imaging Technology 2013; 1: 24-29, doi: 10.13929/j.1003-3289.2013.01.039.

26. Lee JE, Cho KH, Song SK, Kim HJ, Lee HS, Sohn YH, et al. Exploratory analysis of neuropsychological and neuroanatomical correlates of progressive mild cognitive impairment in Parkinson's disease. J Neurol Neurosurg Psychiatry 2014; 85: 7-16, doi: 10.1136/jnnp-2013-305062.

27. Song SK, Lee JE, Park HJ, Sohn YH, Lee JD, Lee PH. The pattern of cortical atrophy in patients with Parkinson's disease according to cognitive status. Mov Disord 2011; 26: 289-296, doi: 10.1002/mds.23477.

28. Zhang J, Zhang YT, Hu WD, Li L, Liu GY, Bai YP. Gray matter atrophy in patients with Parkinson's disease and those with mild cognitive impairment: a voxel-based morphometry study. Int J Clin Exp Med 2015; 8: 15383-15392.

29. Burton EJ, McKeith IG, Burn DJ, Williams ED, O'Brien JT. Cerebral atrophy in Parkinson's disease with and without dementia: a comparison with Alzheimer's disease, dementia with Lewy bodies and controls. Brain 2004; 127: 791-800, doi: 10.1093/brain/awh088.

30. Nagano-Saito A, Washimi Y, Arahata Y, Kachi T, Lerch JP, Evans $A C$, et al. Cerebral atrophy and its relation to cognitive impairment in Parkinson disease. Neurology 2005; 64: 224-229, doi: 10.1212/01.WNL.0000149510. 41793.50.

31. Tam CW, Burton EJ, McKeith IG, Burn DJ, O'Brien JT. Temporal lobe atrophy on MRI in Parkinson disease with dementia: a comparison with Alzheimer disease and dementia with Lewy bodies. Neurology 2005; 64: 861-865, doi: 10.1212/01.WNL.0000153070.82309.D4.

32. Umarova RM, Saur D, Schnell S, Kaller CP, Vry MS, Glauche V, et al. Structural connectivity for visuospatial attention: significance of ventral pathways. Cereb Cortex 2010; 20: 121-129, doi: 10.1093/cercor/bhp086.

33. Rowe JB, Sakai K, Lund TE, Ramsøy T, Christensen MS, Baare WF, et al. Is the prefrontal cortex necessary for establishing cognitive sets? J Neurosci 2007; 27: 1330313310, doi: 10.1523/JNEUROSCI.2349-07.2007.

34. Wolters AF, van de Weijer SCF, Leentjens AFG, Duits AA, Jacobs HIL, Kuijf ML. Resting-state fMRI in Parkinson's disease patients with cognitive impairment: a meta-analysis. Parkinsonism Relat Disord 2019; 62: 16-27, doi: 10.1016/j. parkreldis.2018.12.016.

35. Mihaescu AS, Masellis M, Graff-Guerrero A, Kim J, Criaud $M$, Cho SS, et al. Brain degeneration in Parkinson's disease patients with cognitive decline: a coordinate-based metaanalysis. Brain Imaging Behav 2019; 13: 1021-1034, doi: 10.1007/s11682-018-9922-0.

36. Apostolova LG, Thompson PM. Brain mapping as a tool to study neurodegeneration. Neurotherapeutics 2007; 4: 387-400, doi: 10.1016/j.nurt.2007.05.009.

37. Sabbagh MN, Adler CH, Lahti TJ, Connor DJ, Vedders L, Peterson LK, et al. Parkinson disease with dementia: comparing patients with and without Alzheimer pathology. Alzheimer Dis Assoc Disord 2009; 23: 295-297, doi: 10.1097/WAD.0b013e31819c5ef4.

38. Wang WY, Yu JT, Liu Y, Yin RH, Wang HF, Wang J, et al. Voxel-based meta-analysis of grey matter changes in Alzheimer's disease. Trans/ Neurodegener 2015; 4: 6, doi: 10.1186/s40035-015-0027-z.

39. Ibarretxe-Bilbao N, Ramírez-Ruiz B, Tolosa E, Martí MJ, Valldeoriola F, Bargalló N, et al. Hippocampal head atrophy predominance in Parkinson's disease with hallucinations and with dementia. J Neurol 2008; 255: 1324-1331, doi: 10.1007/s00415-008-0885-8.

40. Xu X, Han Q, Lin J, Wang L, Wu F, Shang $H$. Grey matter abnormalities in Parkinson's disease: a voxel-wise metaanalysis. Eur J Neurol 2019, doi: 10.1111/ene.14132. 